\title{
Venus: key to understanding the evolution of terrestrial planets
}

\author{
Colin F. Wilson ${ }^{1}$ (D) $\cdot$ Thomas Widemann $^{2} \cdot$ Richard Ghail $^{3}$
}

Received: 26 July 2020 / Accepted: 19 May 2021 / Published online: 8 June 2021

(C) The Author(s) 2021

\begin{abstract}
In this paper, originally submitted in answer to ESA's "Voyage 2050" call to shape the agency's space science missions in the 2035-2050 timeframe, we emphasize the importance of a Venus exploration programme for the wider goal of understanding the diversity and evolution of habitable planets. Comparing the interior, surface, and atmosphere evolution of Earth, Mars, and Venus is essential to understanding what processes determined habitability of our own planet and Earth-like planets everywhere. This is particularly true in an era where we expect thousands, and then millions, of terrestrial exoplanets to be discovered. Earth and Mars have already dedicated exploration programmes, but our understanding of Venus, particularly of its geology and its history, lags behind. Multiple exploration vehicles will be needed to characterize Venus' richly varied interior, surface, atmosphere and magnetosphere environments. Between now and 2050 we recommend that ESA launch at least two M-class missions to Venus (in order of priority): a geophysics-focussed orbiter (the currently proposed M5 EnVision orbiter - [1] - or equivalent); and an in situ atmospheric mission (such as the M3 EVE balloon mission - [2]). An in situ and orbital mission could be combined in a single L-class mission, as was argued in responses to the call for L2/L3 themes [3-5]. After these two missions, further priorities include a surface lander demonstrating the high-temperature technologies needed for extended surface missions; and/or a further orbiter with follow-up high-resolution surface radar imaging, and atmospheric and/or ionospheric investigations.
\end{abstract}

Keywords Venus $\cdot$ Space science $\cdot$ Voyage $2050 \cdot$ Planetary mission $\cdot$ Radar $\cdot$ Geochemistry

Colin F. Wilson

colin.wilson@physics.ox.ac.uk

1 Department of Physics, Oxford, UK

2 Paris Observatory, Meudon, France

3 Department of Earth Sciences, Royal Holloway, University of London, Egham, UK 


\section{Introduction}

\subsection{The importance of Venus for comparative planetology}

One of the central goals of planetary research is to find our place in the Universe. Investigating the evolution of solar systems, planets, and of life itself, is at the heart of this quest. The three terrestrial planets in our solar system - Earth, Mars, and Venus show a wide range of evolutionary pathways, and so represent a "key" to our understanding of planets and exoplanets.

Earth and Venus were born as twins - formed at around the same time, with apparently similar bulk composition and the same size. However, they have evolved very differently: the enormous contrast between these planets today challenge our understanding of how terrestrial planets work. The atmosphere is surprising in many ways - its $400 \mathrm{~km} / \mathrm{h}$ winds on a slowly rotating planet; its enormous surface temperature, even though it absorbs less sunlight than does the Earth; its extreme aridity, with sulphuric acid as its main condensable species instead of water. The solid planet, too, is mysterious: its apparent lack of geodynamo and plate tectonics, the uncertainty of its current volcanic state, the apparent young age of much of its surface. How and why does a planet so similar to Earth end up so different?

In an era where we will soon have detected thousands of Earth-sized exoplanets, planetary science must seek to characterise these planets and to explain the diverse outcomes which may befall them. Are these exoplanets habitable? Many efforts have been made to define the edges of the 'habitable zone', i.e. the range of distances from a parent star at which a planet can sustain liquid water on its surface. The inner edge of the habitable zone has been estimated to lie anywhere from $0.5 \mathrm{AU}$ to $0.99 \mathrm{AU}-$ this latter figure, from Kopparapu et al. [6] should be a cause of concern for us Earthdwellers! Detailed study of Venus is indispensable if we are to understand what processes determine the inner edge of the habitable zone.

The habitable zone's boundaries will evolve over a planet's lifetime due to the evolution of the star's output as well as changes in the planet and its atmosphere. There are only three terrestrial planets at which we can study geophysical and evolutionary processes which govern habitability through time: Venus, Earth, and Mars. Exploration of the latter two is firmly established, while in contrast there are, at time of writing in 2019, no confirmed Venus missions after Akatsuki.

\subsection{Context: The state of Venus science after Venus express and Akatsuki}

ESA's Venus Express was a very successful mission. During its science operations, from 2006 to 2014, it made a wealth of discoveries relating to the atmosphere at all altitudes from the surface up to the exosphere. It has mapped cloud motions to reveal wind velocities at different altitudes; it has measured the spatial distributions of key chemical species, and discovered new ones; it has improved our understanding of how unmagnetised Earth-like planets lose water. Despite its atmospheric focus, its most intriguing legacy may be the hints it has provided of current-day volcanic activity.

JAXA's Akatsuki orbiter - previously known as the Venus Climate Orbiter - was launched in 2010, and has been orbiting Venus since 2015 [7]. Its scientific focus is on atmospheric dynamics; it carries a set of cameras using different wavelengths to image 
atmospheric motions at different altitudes. Its observations have revealed new atmospheric waves, providing ever more detailed insights into Venus' atmospheric circulation.

In short, Venus Express and Akatsuki have provided much-needed data for atmospheric dynamics, chemistry, and radiative transfer, and for understanding of its ionosphere and induced magnetosphere. These new data are invaluable for constraining models of how Venus works today. However, the history of Venus still remains enigmatic. In this White Paper, initially submitted in response to ESA's Voyage 2050 call, we propose a new set of investigations that focus on understanding the evolution of Venus through a combination of surface and atmospheric investigations.

Thanks to Venus Express, Europe is at the forefront of Venus research - arguably, this is a situation unmatched in the rest of the Solar System exploration programme. Research groups across Europe have participated in the construction of and analysis from the scientific payload; dozens of researchers have completed doctoral theses based on Venus Express research. Europe is thus well-placed to lead a future Venus mission. ESA can now build on this position, capitalising on investments made in the Earth Observation programme and advanced satellite technologies, to address fundamental questions about the evolution of terrestrial planets and the appearance of life.

\section{Science themes}

\subsection{Geology (interior, tectonism, volcanism, mineralogy, geomorphology)}

The major unknowns in Venus geological science are associated with its resurfacing history and establishing whether it is currently geologically active.

\subsubsection{Resurfacing history}

The age of Venus' surface is poorly known. Unlike Mercury, the Moon, and Mars, Venus has a thick atmosphere that effectively filters out small impactors. As a result, its crater population is limited to a few large craters; there are very few craters with diameters $<20 \mathrm{~km}$, and there are fewer than 1000 craters in total. The observed crater population offers poor constraints on surface ages, allowing a number of different production and resurfacing scenarios. These include catastrophic global lithospheric overturn occurring every 500-700 My [8], equilibrium resurfacing models more similar to those found on Earth [9], as well as many models in between.

The community has used the existing Magellan radar data to attempt to resolve these fundamental conflicts by applying mapping techniques to establish stratigraphic relationships among surface units and structures. NASA's Magellan orbiter, launched in 1989, obtained global radar maps with a spatial resolution of 100-200 m. An important limitation to using radar images for geological mapping is that geological mapping requires the ability to identify distinct rock units, whose formation represent geological processes (e.g., distinct lava flows or sedimentary units). Magellan imagery provides the opportunity to identify some units; for example, it is possible to map lava flow boundaries to high precision in some terrains. But in many, many other cases, the materials being mapped have been affected by later tectonic processes; moreover, the 
highly deformed tessera terrain, thought to be the oldest on Venus, is characterized by overlapping structures whose relationships are ambiguous in currently available observations. Different methods for accommodating this complication have led to widely divergent mapping styles, which in turn have resulted in a range of surface evolution models, mirroring the range of interior evolution models (see e.g. reviews by [10], and [11]). Many remaining debates over, for instance, the sequence and relative timing of tectonic deformation in the complex tesserae cannot be resolved using currently available radar data, because of the limitations represented by the spatial resolution and single polarization of those data.

\subsubsection{Current geological activity}

Venus is thought to have similar internal heat production to Earth, but it is not clear how the internal heat is lost to space. Is heat lost solely by crustal conduction, or does volcanic activity play an important role? Is the loss rate sufficient to maintain an equilibrium or is heat building up in the interior, potentially leading to an episodic resurfacing scenario? Understanding how Venus loses its internal heat is important for understanding both Earth's earliest history and for understanding those exoplanets larger than Earth, both of which share its problem of a buoyant lithosphere; these mechanisms at work may profoundly affect the atmosphere and climate, and prove catastrophic for life.

There are some hints of recent geological activity particularly from Venus Express data $[12,13]$, but these analyses are indirect. A new radar dataset would enable not only better understanding of current surface weathering and alteration processes, which is needed in order to calculate ages for geologically recent changes such as lava flows and dune movements, but would also enable direct searching for surface change.

\subsubsection{Case for next-generation radar}

Because of the extreme surface conditions and opaque clouds on Venus, geological investigation requires orbital remote sensing, with techniques including interferometric synthetic aperture radar (InSAR), gravitometry, altimetry, and infrared observation using nightside infrared windows. The value of radar mapping at Venus was demonstrated by NASA's Magellan orbiter, which obtained global radar maps with a horizontal resolution of 100-200 m and altimetry with a vertical resolution of $100 \mathrm{~m}$. Advances in technology, data acquisition and processing, and satellite control and tracking, mean that the spatial resolutions in the 1-10 $\mathrm{m}$ range are now possible.

This high-resolution radar mapping of Venus would revolutionise geological understanding. Generations of Mars orbital imagery have seen successive order-ofmagnitude improvements, as illustrated above. As imagers progressed from the $50 \mathrm{~m}$ resolution of Viking towards the $5 \mathrm{~m}$ resolution of MOC (on Mars Global Surveyor) and the higher resolutions of HRSC (on Mars Express) and HiRise (on Mars Reconnaissance Orbiter), our conception of Mars as a frozen, inactive planet was followed by hypotheses that geologically recent flow had occurred, to actual detection of current surface changes (e.g. gullies and dune movement) - as illustrated in Fig. 1. For Venus, metre-scale imagery will enable study of Aeolian features and dunes (only two dune 
fields have been unambiguously identified to date on Venus); will enable more accurate stratigraphy and visibility of layering; will constrain the morphology of tesserae enough that their stress history and structural properties can be constrained; will enabled detailed study of styles of volcanism by enabling detailed mapping of volcanic vents and lava flows; and will enable direct search for surface changes due to volcanic activity and Aeolian activity. Metre-scale resolution would even enable a search for changes in rotation rate due to surface-atmosphere momentum exchange, which could constrain internal structure [14].

The revolutions in radar performance go beyond just spatial resolution. Differential InSAR allows surface change detection at centimetre scale. This technique has been used to show surface deformations after earthquakes and volcanic eruptions on Earth (see Fig. 2); similar results on Venus could provide dramatic evidence of current volcanic or tectonic activity.

The maturity of InSAR studies on Earth is such that data returned from Venus can confidently be understood within a solid theoretical framework developed from coupled terrestrial InSAR data and ground-truth observations, even without groundtruth data on Venus. Note that the radar spatial resolution may be high enough to permit identification of Venera and Pioneer Venus landers, which serves as some ground truth even before a new generation of landers is taken into account. Radar mapping with different polarisation states constrains surface roughness and dielectric properties; mapping at different look angles and different wavelengths will provide further new constraints on surface properties.

The proximity of Venus to Earth, the relatively calm (if extreme) surface conditions and lack of water, the absence of a large satellite and its moderately well-known geoid and topography, all help to ease the technical demands on the mission. Europe has developed a number of world-leading radar systems and could, for example, adapt a GMES Sentinel-1 or NovaSAR-S modular array antenna for use at Venus, providing higher-resolution imagery, topography, geoid, and interferometric change data that will revolutionise our understanding of surface and interior processes [16].

In addition to radar techniques, the surface can also be observed by exploiting nearinfrared spectral window regions at wavelengths of $0.8-2.5 \mu \mathrm{m}$. On the nightside of

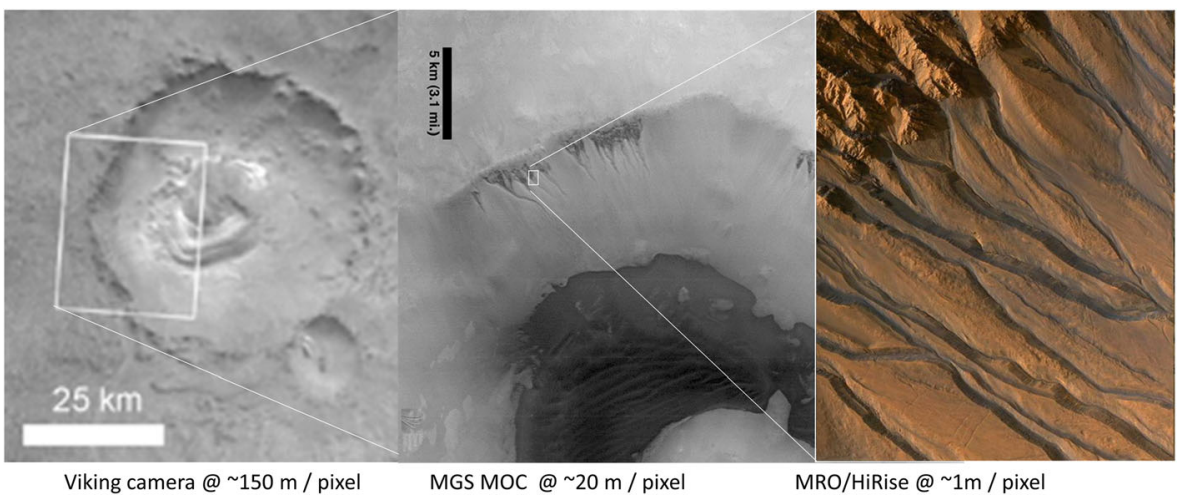

Fig. 1 Images of the same region of Mars illustrate the revolutions in understanding which are enabled by increasing spatial resolution by an order of magnitude, particular for surface processes. Quoted pixel resolution is that of the displayed image used rather than the full resolution of the original. Image credits: NASA/JPL 


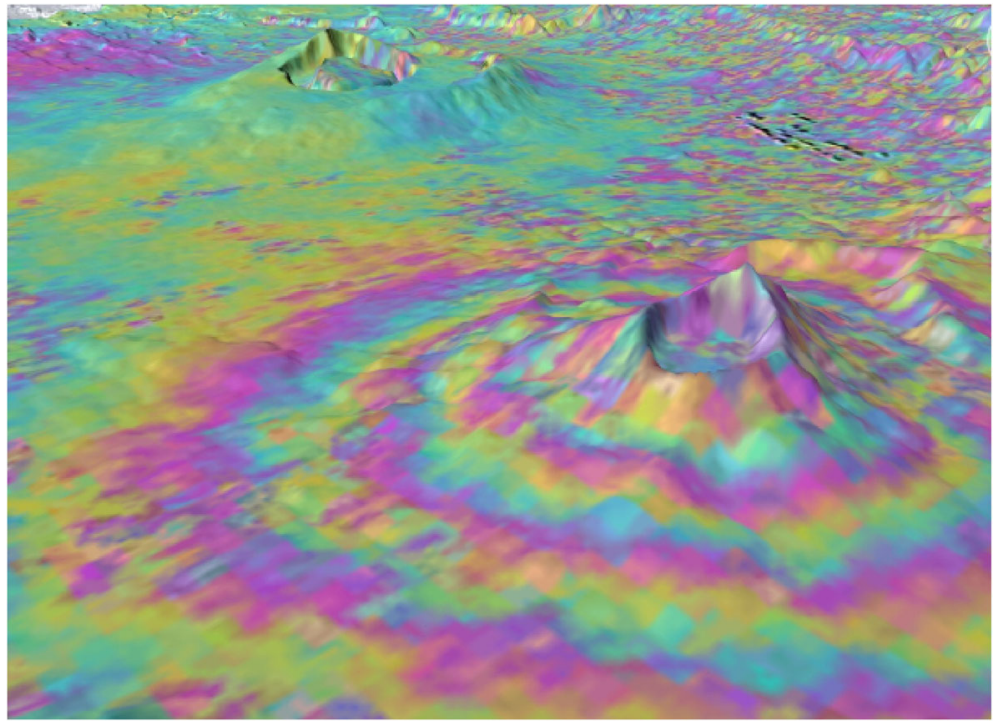

Fig. 2 Differential InSAR revealed altimetry changes in this volcano in Kenya which previously had been thought to be dormant. [[15]; observations from Envisat ERS-1 \& ERS-2]

Venus, thermal emission from the surface escapes to space in some of these spectral windows, allowing mapping of surface thermal emissivity, as demonstrated by the VIRTIS instrument on Venus Express [17]. A new instrument optimised for this observation could map mineralogy and also monitor the surface for volcanic activity [18].

\subsubsection{Case for Venus in situ geological investigations}

The Venera and Vega missions returned data about the composition of Venus surface materials, but their accuracy is not sufficient to permit confident interpretation. The Venera and Vega analyses of major elements (by X-ray fluorescence, XRF) did not return abundances of $\mathrm{Na}$, and their data on $\mathrm{Mg}$ and $\mathrm{Al}$ are little more than detections at the $2 \sigma$ level. Their analyses for K, U, and Th (by gamma rays) are imprecise, except for one (Venera 8 ) with extremely high $\mathrm{K}$ contents $\left(\sim 4 \% \mathrm{~K}_{2} \mathrm{O}\right)$ and one (Venera 9$)$ with a non-chondritic $\mathrm{U} / \mathrm{Th}$ abundance ratio. The landers did not return data on other critical trace and minor elements, like $\mathrm{Cr}$ and $\mathrm{Ni}$. In addition, the Venera and Vega landers sampled only materials from the Venus lowlands - they did not target sites in any of the highland areas, the coronae, tesserae, nor the unique plateau construct of Ishtar Terra. Currently available instruments could provide much more precise analyses for major and minor elements, even within the engineering constraints of Venera-like landers.

A new generation of geologic instrumentation should be brought to the surface of Venus, including Raman/LIBS (Laser Induced Breakdown Spectroscopy) and XRF/ $\mathrm{XRD}$ (X-ray diffraction); this would allow mineralogical, as well as merely elemental, composition. Such precise analyses would be welcome for basalts of Venus' lowland plains, but would be especially desirable for the highland tesserae and for Ishtar Terra. The tesserae are thought to represent ancient crust that predates the most recent 
volcanic resurfacing event and so provide a geochemical look into Venus' distant past. Ishtar Terra, too, may be composed (at least in part) of granitic rocks like Earth's continental crust, which required abundant water to form. Coronae samples will reveal how magmatic systems evolved on Venus in the absence of water but possibly in the presence of $\mathrm{CO}_{2}, \mathrm{SO}_{2}$, or other volatiles. Surface geological analysis would benefit from high temperature drilling/coring and sample processing capabilities, although further investigation will be needed to assess the extent to which this can be within the scope of even an L-class mission opportunity.

Long-lived stations would provide essential seismological and meteorological data. The technological barriers to such missions are twofold: (1) the high temperature environment of the Venus surface, too hot for silicon electronics, and (2) the lack of sunlight at the surface, making solar power unviable. As has been discussed by Wilson et al. [19] and Kremic et al. [20], recent advances in high-temperature electronics have made long-duration uncooled landers a possibility which could be explored in the coming decades; this requires continued investment in the development of the electronics, in their packaging, and in their environmental qualification in Venus conditions. Power for long-duration landers on the surface could come from primary molten salt batteries, for a first generation of landers. Second-generation long-life landers could be powered by radioisotope thermoelectric generators (RTGs) or even wind powerboth of which would require technology development.

Descent imaging has not yet been performed by any Venus lander. Descent imaging of any landing site would be useful, particularly so for the tessera highlands where it would reveal the morphology of the highland surfaces and yield clues as to what weathering processes have been at work in these regions. Multi-wavelength imaging in near-infrared wavelengths would yield compositional information to provide further constraints on surface processes. In particular, descent imaging can establish whether near-surface weathering or real compositional differences are the root cause for nearinfrared emissivity variations seen from orbit [21] - providing important ground truth for these orbital observations.

Profiles of atmospheric composition in the near-surface atmosphere would reveal which chemical cycles are responsible for maintaining the enormously high carbon dioxide concentrations in the atmosphere, and would also reveal details about surfaceatmosphere exchanges of volatiles. Several mechanisms have been invoked for buffering the observed abundance of carbon dioxide, including the carbonate [22] or pyritemagnetite [23] buffer hypotheses. Measurements of near-surface abundances and vertical gradients of trace gases, in particular $\mathrm{SO}_{2}, \mathrm{H}_{2} \mathrm{O}, \mathrm{CO}$, and OCS, would enable discrimination between different hypotheses. Correlating these data with lander and orbiter observations will reveal how important and widespread the sources and sinks of these species might be.

Measurement of the temperature, pressure, and $\mathrm{N}_{2}$ abundance in the lowest scale height is also essential. Only one Venus descent probe, Vega 2 in 1984, reported temperature and pressure profiles all the way down to the surface; and the gradient of its temperature profile in the lowest few km appears far more convectively unstable than is thought to be physically possible. At the altitudes where this occurs, carbon dioxide is in a supercritical fluid regime, and it has been suggested that carbon dioxide and nitrogen are separating due to exotic supercritical processes [24]. This effect would have major implications for our understanding of high pressure atmospheres 
everywhere, from the gas giants to exoplanets. High-accuracy measurements of pressure, temperature, and $\mathrm{N}_{2}$ abundance down to the surface would resolve this intriguing question.

\subsection{Planetary evolution as revealed by isotope geochemistry}

Geology is a powerful witness to history, but it does not provide answers about evolution in the time before the formation of the oldest rocks, which on Venus are thought to have formed only about a billion years ago. For constraints on earlier evolution, we must turn to isotope geochemistry.

Radiogenic noble gas isotopes provide information about the degassing history of the planet. ${ }^{40} \mathrm{Ar}$, produced from the decay of long-lived ${ }^{40} \mathrm{~K}$, has been continuously accumulated over $>4$ billion years and so its current abundance constrains the degree of volcanic/tectonic resurfacing throughout history. ${ }^{129} \mathrm{Xe}$ and ${ }^{130} \mathrm{Xe}$ are produced from the now extinct ${ }^{129} \mathrm{I}$ and ${ }^{244} \mathrm{Pu}$ respectively within the first $100 \mathrm{Ma}$ of the Solar System's history. The depletion of these isotopes in the atmospheres of Mars and Earth reveals that these two planets underwent a vigorous early degassing and blow-off, although the mechanisms of this blow-off and of subsequent deliveries of materials from comets and meteorites vary according to different scenarios. Neither the bulk Xe abundance nor the abundances of its eight isotopes have ever been measured at Venus. Measurements of these abundances would provide entirely new constraints on early degassing history. ${ }^{4} \mathrm{He}$, produced in the mantle from long-lived $\mathrm{U}$ and $\mathrm{Th}$ decay, has an atmospheric lifetime of only a few hundred million years before it is lost by escape to space. Therefore its current atmospheric abundance provides constraints on recent outgassing and escape rates within the last $10^{8}-10^{9}$ years.

Non-radiogenic noble gas isotopes provide information about acquisition and loss of planet-forming material and volatiles. Venus is less depleted in Neon and Argon isotopes than are Earth and Mars, but its $\mathrm{Xe}$ and $\mathrm{Kr}$ isotopic abundances are still unknown: Xe isotopic abundances have not yet been measured, and past measurements of $\mathrm{Kr}$ abundance vary by an order of magnitude, providing little useful constraint. The significant fractionation of xenon on Earth and Mars can be attributed to massive blowoffs of the initial atmospheres in the period after the radiogenic creation of xenon from its parent elements, $\sim 50-80 \mathrm{Myr}$ after planet formation. However, it could also be that the fractionation is reflecting that of source material delivered late in planetary formation, perhaps from very cold comets. If Venus has the same xenon fractionation pattern as Earth and Mars, this would support the idea that a common source of fractionated xenon material was delivered to all three planets, and weaken the case that these reflect large blowoffs. If we see a pattern with less Xe fractionation then that would support the blow-off theory for Earth and Mars. Considered together with other non-radiogenic isotopic abundances, this allows determination of the relative importance of EUV, impact-related or other early loss processes. These measurements would also allow tighter constraints on how much of the gas inventory originated from the original accretion disc, how much came from the solar wind, and how much came later from planetesimals and comets. Late impacts such as the Earth's moon-forming impact also have an effect on noble gas isotopic ratios so can also be constrained through these measurements. 
Light element isotopic ratios, namely $\mathrm{H}, \mathrm{C}, \mathrm{O}, \mathrm{N}$, etc., provide further insights into the origin and subsequent histories of planetary atmospheres. Measurement of ${ }^{20} \mathrm{Ne} /$ ${ }^{21} \mathrm{Ne} / 22 \mathrm{Ne}$ and/or of ${ }^{16} \mathrm{O} /{ }^{17} \mathrm{O} /{ }^{18} \mathrm{O}$ would enable determination of whether Earth, Venus, and Mars came from the same or from different parts of the protoplanetary nebula, i.e. are they are truly sibling planets of common origin. Venus' enhanced deuterium to hydrogen ratio, 150 times greater than that found on Earth, suggests that hydrogen escape has played an important role in removing water from the atmosphere of Venus, removing more than a terrestrial ocean's worth of water during the first few hundred million years of the planet's evolution [25]. ${ }^{14} \mathrm{~N} /{ }^{15} \mathrm{~N}$ ratios have been found to vary considerably in the Solar System, with the solar wind, comets, and meteorites all exhibiting isotopic ratios different from the terrestrial values; it also is affected by preferential escape of ${ }^{14} \mathrm{~N}$. Measurement of the nitrogen isotopic ratio therefore helps establish whether the gas source was primarily meteoritic or cometary, and constrains the history of escape rates.

Taken together, and as illustrated in Fig. 3, measurements of these isotopic abundances on Venus, Earth, and Mars are needed to provide a consistent picture of the formation and evolution of these planets and their atmospheres, and in particular the history of water on Venus. Early Venus would have had an atmosphere rich in carbon dioxide and water vapour, like that of Hadean Earth. Hydrodynamic escape from this early steam atmosphere would have been rapid - but would it have been rapid enough to lose all of Venus' water before the planet had cooled enough to allow water to condense? If Venus did have a liquid water ocean, how long did this era persist before the runaway greenhouse warming 'ran away', with the oceans evaporating and the resulting water vapour being lost to space? The nature of early escape processes is as yet too poorly constrained to answer these questions. An early Venus with a liquid water ocean would have arguably have been more Earth-like than was early Mars and could have taken steps towards the development of life. A habitable phase for early Venus would have important consequences for our understanding of astrobiology and the habitable zones of exoplanets.

This section of the paper has argued that new measurements of isotopic abundances are needed. A few isotopic ratios, notably $\mathrm{D} / \mathrm{H}$, can be measured from orbit, and indeed orbital measurements allow a comprehensive understanding of how they may vary in space and time. However, most of the isotopic abundances in question, particularly the valuable abundances of noble gases isotopes, cannot be measured remotely and require measurement in situ. Noble gas isotopes are non-reactive so will be well-mixed throughout the atmosphere; a measurement of their abundances anywhere below the homopause altitude of some 120-130 km will be representative of the whole lower atmosphere. Some isotopic abundances were measured by Venera and Pioneer Venus entry probes, but the low sensitivity of 1970 s technologies used in these measurements means that many key species abundances were not measured at all, or not measured with an accuracy which provides useful constraints on Venus planetary evolution. New measurements, using modern spacecraft mass spectrometers in which European groups have considerable expertise, can be made either from a classical Venera-style descent probe, which descends to the surface within one hour or so, or from a long-lived platform dwelling for days or weeks in the cloud layer taking advantage of the benign ambient temperatures found there to make repeated measurements. 


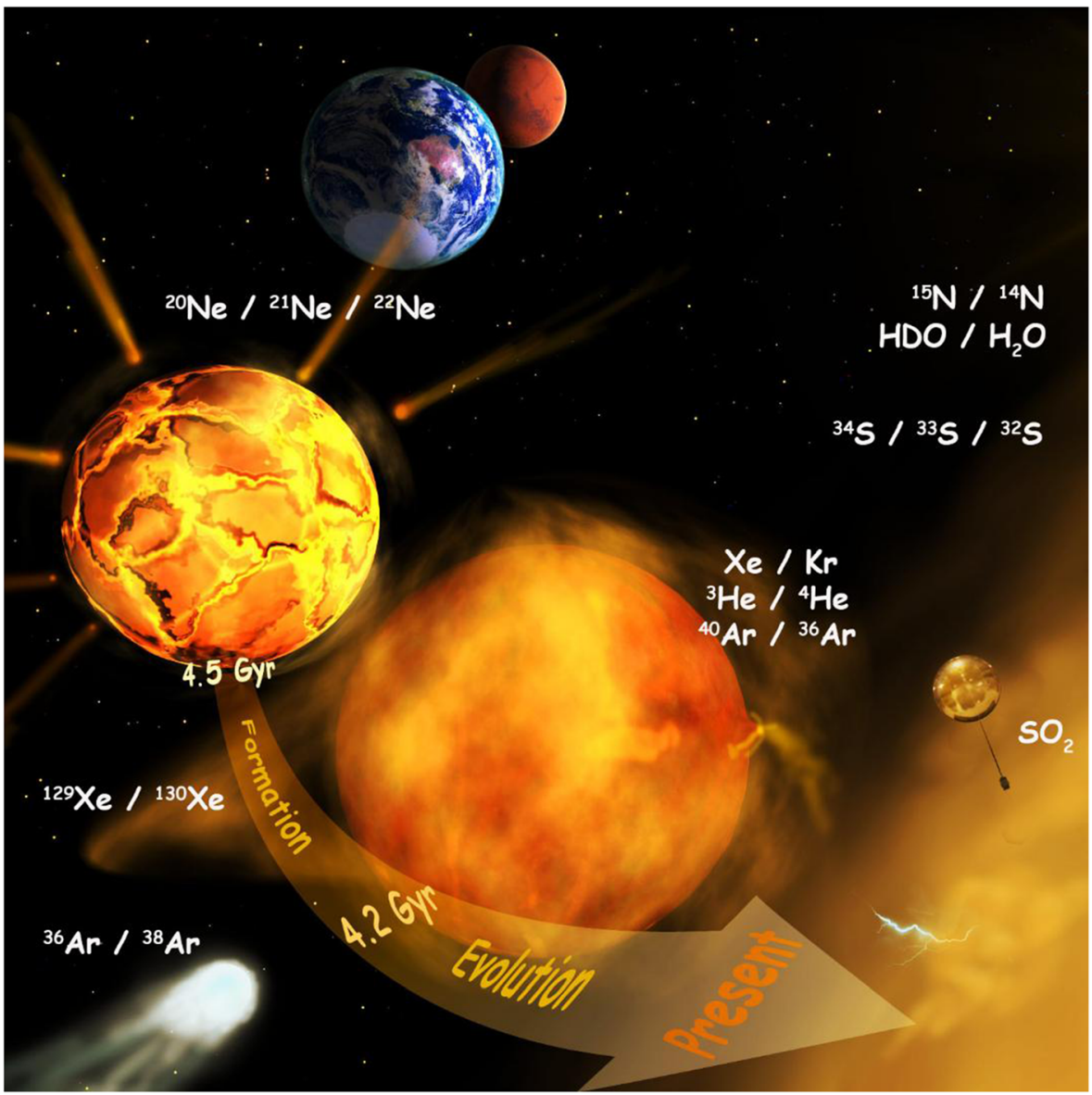

Fig. 3 Isotopic ratios provide keys to constrain planetary origins ( $\mathrm{Ne}$ isotopes), early atmospheric loss processes (non-radiogenic $\mathrm{Xe}, \mathrm{Kr}, \mathrm{Ar}$ ), late impact scenarios, recent mantle outgassing and late resurfacing, and escape of water. Modified from [26]

More detailed treatments of Venus isotope geochemistry goals and interpretation can be found in Chassefière et al. [27] and Baines et al. [26].

\subsection{Atmospheric science (dynamics; chemistry and clouds; structure and radiative balance)}

The terrestrial planets today exhibit strikingly different climates. Study of the fundamental processes at work on these three planets will lead to a deeper understanding of how atmospheres work, and of how climates evolve.

\subsubsection{Dynamics and thermal structure}

One of the crucial factors determining planetary habitability is the redistribution of heat around the planet. The solid planet of Venus rotates only once every 243 Earth days but the atmosphere above exhibits strong super-rotation, circling the planet some $40-50$ 
times faster than the solid planet below. General Circulation Models (GCMs) are now able to reproduce super-rotation, but are very sensitive not only to model parameters but also to the details of how those models operate. Efforts to improve modelling of the Venus atmosphere have led to improvements in how Earth GCMs handle details like conservation of angular momentum and temperature dependent specific heat capacities [28]. Exchanges of momentum between surface and atmosphere, an important boundary condition for the atmospheric circulation, depend sensitively on the thermal structure in the lowest $10 \mathrm{~km}$ of the atmosphere. Many probes experienced instrument failure at these high temperatures so the atmospheric structure in the lowest parts of the atmosphere is not well known. The atmospheric circulation is driven by solar absorption in the upper cloud; most of this energy absorption is by an as-yet-unidentified UV absorber, which is spatially and temporally variable. Efforts to identify this UV absorber by remote sounding have been inconclusive, so in situ identification will be necessary.

Most of our knowledge of the wind fields on Venus has been gained by tracking imaged cloud features, or by tracking descent probes. Tracking cloud features returns information about winds at altitudes from $48 \mathrm{~km}$ to $70 \mathrm{~km}$ altitude, depending on the wavelength used. However, it is not clear at what altitude to assume that the derived cloud vectors apply; the formation mechanism for the observed contrasts is not known so it cannot be ascertained to what extent the derived velocity vectors represent true air motion rather than the product of, for example, wave activity. Furthermore, with the exception of recent observations from Akatsuki's Longwave Infrared (LIR) Camera, observations on the day- and night-sides of Venus are obtained using different wavelengths, referring to different altitudes on day- and night-sides, so global averages of wind fields cannot be obtained by wind tracking, frustrating attempts to understand global circulation.

Direct measurement of mesospheric wind velocities (or at least their line-of-sight components) from orbit can be achieved by using Doppler sub-millimetre observations, Doppler LIDAR, or other such instruments, and this would provide invaluable constraints for circulation models. Tracking of descent probes will yield direct measurement of the vertical profile of horizontal winds in the deep atmosphere, which will provide important constraints on the mechanisms of super-rotation. Balloon elements are ideal for measuring vertical wind speeds but also provide information about wave and tidal activity at near-constant altitude.

\subsubsection{Chemistry and clouds}

Venus has an enormous atmosphere with many complex chemical processes at work. Processes occurring near the surface, where carbon dioxide becomes supercritical and many metals would melt, are very different from those at the mesopause where temperatures can be below $-150{ }^{\circ} \mathrm{C}$, colder than any found on Earth. A diversity of observing techniques is clearly required to understand this diversity of environments. While chemical processes in the mesosphere and lower thermosphere were studied in depth by Venus Express, processes in the clouds and below are very difficult to sound from orbit and require in situ investigation.

The dominant chemical cycles at work in Venus's clouds are those linking sulphuric acid and sulphur dioxide: Sulphuric acid is photochemically produced at cloud-tops, 
has a net downwards transport through the clouds, and then evaporates and then thermal dissociates below the clouds; this is balanced by net upwards transport through the clouds of its stable chemical precursors $\left(\mathrm{SO}_{2}\right.$ and $\left.\mathrm{H}_{2} \mathrm{O}\right)$. Infrared remote sensing observations of Venus can be matched by assuming a cloud composition entirely of sulphuric acid mixed with water, but Vega descent probe XRF measurements found also tantalising evidence of $\mathrm{P}, \mathrm{Cl}$, and even $\mathrm{Fe}$ in the cloud particles [29]. If confirmed these measurements would provide important clues as to exchanges with the surface: are these elements associated with volcanic, Aeolian, or other processes? The UV-blue portion of the spectrum, too, shows evidence of an as-yet-unidentified absorber in the clouds besides sulphuric acid, which could be polysulphur $\left(\mathrm{S}_{3}, \mathrm{~S}_{4}, \mathrm{~S}_{\mathrm{x}}\right)$, iron chloride, or a number of other possibilities. Chemical and dynamical models of the Venus clouds have, understandably, been held back by an ignorance of the detailed composition of the clouds and hazes. An in situ chemical laboratory floating in the clouds, or multiple descent probes, is needed to address cloud composition and chemical processes.

As to lower atmosphere chemistry, it is poorly understood because the rapidly falling descent probes had time to ingest and fully analyse only a small number of atmospheric samples during their brief descent. Sub-cloud hazes were detected by several probes but their composition is unknown. Ground- and space-based observations in near-infrared window regions permit remote sounding of a few major gases, but many minor species which may play important catalytic or intermediate roles in chemical cycles are difficult or impossible to probe remotely. Mapping spatial variation of volcanically gases could be a direct way of finding active volcanism on Venus; this can be achieved using spectroscopy in nightside near-infrared, as is being developed for the EnVision M5 mission [30]. Further to this, measurement from a descent probe of near surface chemical abundances and their vertical profiles will permit determination of whether there are active surface-atmosphere exchanges taking place and of what surface reactions are buffering the atmospheric composition.

\subsubsection{Thermal structure and radiative fluxes}

The cloud layer of Venus is highly reflective so Venus presently absorbs less power from the Sun than does the Earth. Its high surface temperature is instead caused by its enormous greenhouse warming effect, caused by carbon dioxide, water vapour, and other gases. Its clouds, too, have a net warming effect because they prevent thermal fluxes from escaping the deep atmosphere.

1-D radiative and radiative-convective models for the determination of equilibrium climate are now widespread as researchers worldwide attempt to determine the likely climate of exoplanets. Venus offers a proving ground for these models much closer to home, one where the conditions are much better known than on exoplanets. Radiative transfer calculations on Venus are difficult: uncertainties in the radiative transfer properties of carbon dioxide at high temperatures and pressures are the main unknown, particularly in the middle- and far- infrared where there are no spectral window regions to allow empirical validation. As on Earth, clouds play an important role, reflecting away sunlight but also trapping upwelling infrared radiation. The state-of-the-art Venus radiative balance models are still mainly $1-\mathrm{D}$ models representing an average over the whole planet. However, we now know that the clouds are very variable; the vertically integrated optical thickness (as measured at $0.63 \mu \mathrm{m}$ ) can vary by up to $100 \%$ [31] and 
the vertical structure of clouds varies strongly with latitude. In-situ measurements of cloud properties with co-located radiative flux measurements are needed to determine the diversity of cloud effects on the global radiative balance.

\section{Mission elements}

The long list of scientific investigations outlined above cannot all be investigated from a single spacecraft: a range of different platform types, as illustrated in Fig. 4, can be considered.

A satellite in low, near-circular polar orbit is required for radar mapping, and for LIDAR and Doppler sub-mm measurement of wind speeds. Wide-angle cameras like the MARCI camera on Mars Reconnaissance Orbiter can be used to obtain continuous imaging coverage of UV cloud-top features. Recent examples of proposed low circular Venus orbiters include the Envision M5 radar mapper [1], VERITAS radar mapper [32], the RAVEN radar mapper [33], MuSAR radar mapper [34], and the Vesper submm sounding orbiter [35].

A satellite in a highly elliptical orbit can provide synoptic views of an entire hemisphere at once. One example of this is Venus Express, whose polar apocentre allows it to study the vortex circulation of the South polar region; a second example is

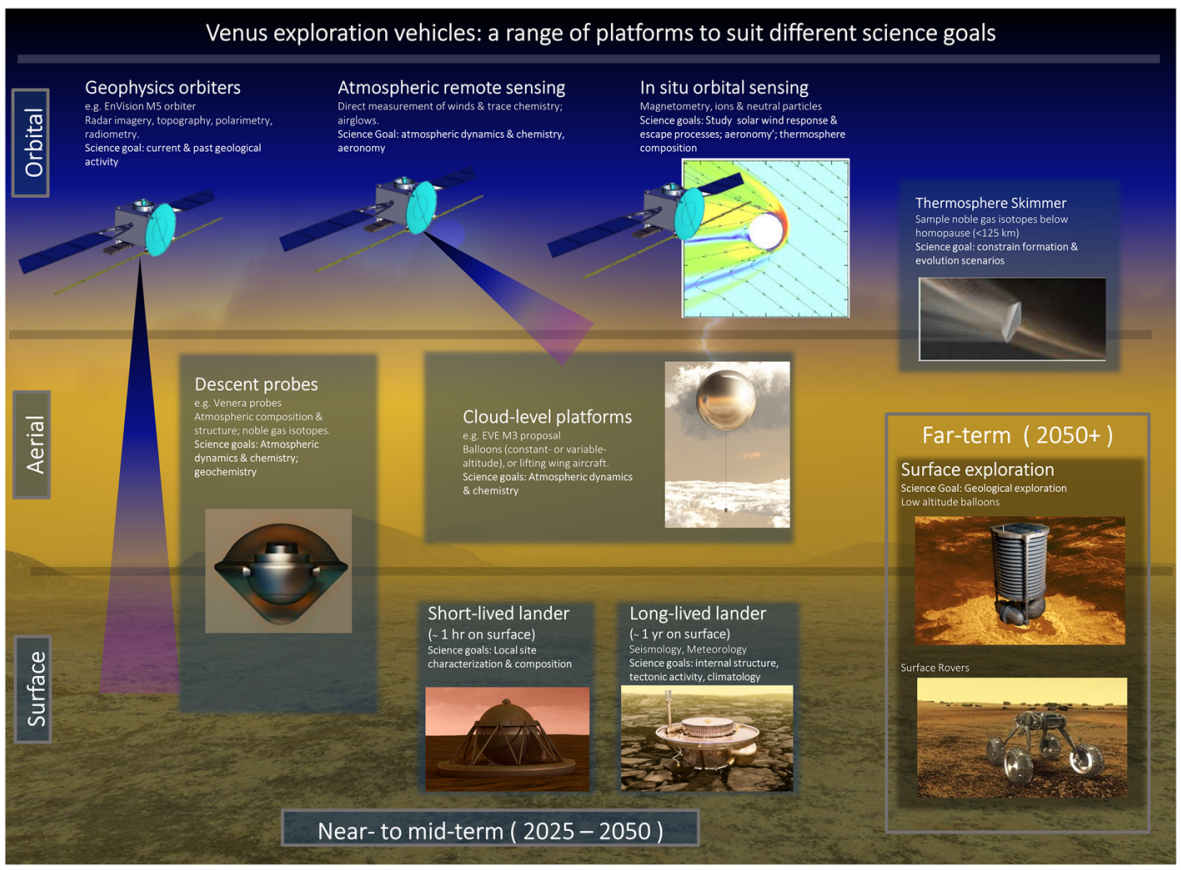

Fig. 4 High-priority Venus questions can be addressed by a broad range of mission concepts using surface, aerial, and orbital platforms. While many missions can be implemented in the near term using existing capabilities, investments in new technologies will enable long term surface science as well as missions that take advantage of mobility in the surface, near-surface, and atmospheric environments. Venus exploration will be undertaken by a range of international agencies; for the period 2030-2050, we recommend that ESA focus on launching at least one geophysics orbiter and one cloud-level balloon 
the nearly equatorial 40-h orbit of Japan's Akatsuki orbiter, which allows it to dwell over low-latitude cloud features for tens of hours at a time. Furthermore, the large range of altitudes reached by satellites in such orbits is useful for in situ studies of thermosphere and ionosphere and thus for studies of solar wind interaction and escape.

Balloons are ideally suited for exploring Venus because they can operate at altitudes where pressures and temperatures are far more benign than at the surface. Deployment of two small balloons at $\sim 55 \mathrm{~km}$ altitude, in the heart of the main convective cloud layer, was successfully demonstrated by the Soviet Vega mission in 1984. At this altitude, the ambient temperature is a comfortable $\sim 20^{\circ} \mathrm{C}$ and the pressure is $0.5 \mathrm{~atm}$. The main environmental hazard is the concentrated sulphuric acid which makes up the cloud particles; however, the cloud abundance is relatively low (peak cloud mass loadings on the order of only $30 \mathrm{mg} / \mathrm{m}^{3}$ as reported in Knollenberg and Hunten [36], similar to those found in cirrus clouds on Earth), and its chemical effects can be mitigated by choosing appropriate materials for external surfaces. Balloons at this altitude can take advantage of the fast super-rotating winds which will carry the balloon all the way around the planet in a week or less (depending on latitude and altitude). Horizontal propulsion (with motors) is not usually viable because of power requirements and the difficulty of countering the fast $(250 \mathrm{~km} / \mathrm{h})$ zonal windspeed. A cloudlevel balloon is an ideal platform for studying interlinked dynamical chemical and radiative cloud-level processes. It also offers a thermally stable long-lived platform from which measurements of noble gas abundances and isotopic ratios can be carefully carried out and repeated if necessary (in contrast to a descent probe, which offers one chance for making this measurement, in a rapidly changing thermal environment).

Balloons can be used to explore a range of altitudes. Operation in the convectively stable upper clouds, above $63 \mathrm{~km}$, would be optimal for identification of the UV absorber, but the low atmospheric density leads to a relatively small mass fraction for scientific payload. Operation below the main cloud deck at $40 \mathrm{~km}$, has been proposed by Japanese researchers, with a primary goal of establishing wind fields below the clouds. Balloons can also be used to image the surface, if they are within the lowest 1$10 \mathrm{~km}$ of the atmosphere, but high temperatures here require exotic designs such as metallic bellows which are beyond the scope of this paper. An intriguing possibility for revealing winds in the lower atmosphere is to use passive balloons, reflective to radio waves, which could be tracked by radar - this possibility should be studied further if a radar orbiter + entry probes architecture were to be studied further.

Descent probes provide vertical profiles of composition, radiation, chemical composition as a function of altitude, and enable access to the surface. Science goals for a descent probe include: cloud-level composition and microphysical processes; nearsurface composition, winds, and temperature structure; surface composition and imaging; and noble gas abundances and isotopic ratios. If the scientific focus of the probe is measurements at cloud level then a parachute may be deployed during the initial part of the entry phase in order to slow the rate of descent during the clouds. This was carried out, for example, by the Pioneer Venus Large Probe. Alternatively, if the main focus of the probe is measurements in the lower atmosphere or surface then the probe may dispense with a parachute completely. Descent imagery of impact/landing site at visible wavelengths will be invaluable for contextualising surface results; Rayleigh scattering limits the altitudes from which useful surface imagery can be obtained to $\sim 1 \mathrm{~km}$ if imaging in visible wavelengths, or to $10 \mathrm{~km}$ if imaging at $1 \mu \mathrm{m}$ wavelength [37]. 
Landers must cope with the harsh conditions of $\sim 450{ }^{\circ} \mathrm{C}$ at the surface of Venus. Venera-style landers use only thermal inertia and thermal insulation to keep a central electronics compartment cool, allowing operation times of only hours (see e.g. VeneraD mission, [38]). The principal science payload of a such a lander would include surface imagers and non-contact mineralogical sensors such as a Gamma spectrometer with Neutron activation, capable of measuring elemental abundances of U, Th, K, Si, $\mathrm{Fe}, \mathrm{Al}, \mathrm{Ca}, \mathrm{Mg}, \mathrm{Mn}, \mathrm{Cl}$ [39], and/or Raman/LIBS [40]. Inclusion of surface sample ingestion via a drill/grinder/scoop would allow further analysis techniques (e.g. mass spectroscopy; X-ray fluorescence (XRF) spectroscopy) but would require significant technology development. Gamma- and XRF spectroscopy was performed on Venera and Vega landers, but modern equivalents of these instruments would provide much improved accuracy; also, repeating the composition analyses at a tessera region (not before sampled) would reveal whether these tessera regions are chemically differentiated from the lava plains where previous analyses have been conducted.

As discussed above, long-lived landers, using high temperature electronics so as to not require any active cooling, would not only provide key meteorological and seismic measurements, but would also serve as precursors for more capable seismometry stations (more like Insight in their capability) and eventual surface rovers - which we envisage as post-2050 developments. A recent review of the state of the art and possibilities for near-future technology demonstrators can be found in Wilson et al. [19].

\section{A strawman L - class mission architecture}

A Large mission to Venus should include both orbital and in situ science measurements. One possible strawman mission concept which could address this theme would be a combination of an orbiter, a cloud-level balloon platform, and (optionally) a Russian descent probe/lander. As a strawman payload, we suggest the balloon element be modelled on the 2010 EVE M3 proposal [2]. The radar-equipped orbiter may be based on EnVision M3, M4, or M5 proposals [1, 41]. Finally, the landing probe envisaged is based on the lander component of the Venera-D mission [38].

It is very important to have multiple mission elements working together simultaneously at Venus. An orbiter is necessary both to increase vastly the volume of data returned from the in situ elements, but also to place those in situ measurements into atmospheric and geological context - including, literally, determining the position of the in situ element, which is particularly important for balloons when they are not visible from Earth. The in situ measurements are required to measure parameters, like noble gas abundances and surface mineralogy, which cannot be determined from orbit. The whole mission is greater than the sum of its parts. This has been amply demonstrated by the constellation of missions at Mars, and by the Cassini/Huygens collaboration at Titan.

Not all of these mission elements need be provided by ESA; there is ample scope for international co-operation in creating this mission architecture. In particular, Russia has unequalled heritage in providing Venus descent probes from its Venera and Vega descent probes, and will gain new heritage from its Venera-D lander, planned for the coming decade. A range of mission proposals have been developed in the USA for 
orbiters, balloons, and descent probes, from Discovery-class to Flagship-class, many of which could form parts of a joint NASA-ESA exploration programme should a highlevel agreement be reached. Japan has an active Venus research community, with its Akatsuki (Venus Climate Orbiter) spacecraft still in flight, and has developed prototypes for a Venus sub-cloud balloon [42]. After its successful moon and Mars missions, ISRO has been developing a Venus orbiter which may launch as soon as 2023 [43] but this, at time of writing, is still not confirmed. Israel's TECSAR satellites enable 1-m scale radar mapping with a $300 \mathrm{~kg}$ satellite; in the frame of future ESA-Israel agreements, collaborations on Venus radar could be fruitful. In this time frame collaborations with China are also feasible.

These could be launched as a stack on a single launcher, or it may prove convenient to use separate launchers, for example in order to insert the orbiter into a low circular orbit before the arrival of the in situ elements for optimal data relay and contextual remote sounding for the in situ measurements.

This scenario, Orbiter + balloon + Descent Probe, was proposed to ESA in 2007 in response to the M1/M2 mission Call for Ideas as a joint European Russian mission, with a European-led orbiter and balloon, and a Russian descent probe [44]. Referred to as EVE 2007, the entire mission was to be launched on a single Soyuz launch, however subsequent studies revealed that this scenario was not consistent with a single Soyuz launcher and would be more consistent with an L-class rather than an M-class opportunity. The Venus Flagship Mission being studied by NASA in 2019-2020 provides another example of a large mission of this architecture [45].

\section{Technology developments needed}

Much of the technology required for Venus missions in the Voyage 2050 period already exists at a high technology readiness level, but further technology development both for spacecraft technologies and for science payload technologies would be useful to maximise science return and de-risk mission aspects, and to pave the way for future surface missions.

Many of the mission-enabling technologies required for Venus exploration are shared with other targets. Aerobraking/aerocapture would improve $\Delta v$ and mass budgets for Venus orbiters. Further improvement in deep space communications, including development of Ka-band or optical communications, would provide increased data return from orbiters which would be particularly useful for the large volumes of data generated by high-resolution radar instrumentation at Venus: data rates reaching $100 \mathrm{Mbps}$ or above are needed to return of Earth-like radar observations for a large fraction of the planet. High speed entry modelling, thermal protection systems, and parachute development will all be useful for Venus entry probes. Nuclear power systems are not necessary for the strawman Venus mission elements described here, but would enable longer lifetimes and increased nightside operations for the balloon element of the mission, and will be necessary for long-lived surface stations (in the even more distant future) due to the scarcity of sunlight reaching the Venus surface and long night-time duration.

Two technology areas specific to Venus exploration are balloon technology, and high-temperature $\left(\sim 450{ }^{\circ} \mathrm{C}\right)$ components. The balloons proposed in this strawman 
mission (based on the EVE M3 proposal) are helium superpressure balloons, which are designed to float at constant altitude. Although ESA has little familiarity with this technique, thousands of helium superpressure balloons have been launched on Earth, and two were successfully deployed on Venus in 1985 as part of the Russian Vega programme, so this is a mature technology. Air-launching of balloons - deploying them from a probe descending under parachute - was achieved by the Russian Vega balloons, was demonstrated by CNES in Vega development programmes, and demonstrated recently by JPL engineers in their own Venus balloon test programme; nevertheless, a new demonstration programme in Europe would be required to obtain recent European experience for this technology. Balloon envelope design and sulphuric acid resistance verification such as those carried at NASA/JPL [46] would also be valuable to conduct in Europe. Feasibility studies on other forms of aerial mobility, including controllable-altitude balloons, phase change fluid balloons, $<5 \mathrm{~kg}$ microprobes and fixed wing aircraft, would also be useful for expanding the possibilities of long-term future exploration programmes. Data downlink and location determination present particular challenges for Venus balloons; addressing this with medium- or high-gain electronically steerable phased array antennae would increase the scientific return from these mission elements. A programme of investment in all of these systems, in collaboration with national agencies, would be valuable.

High temperature technologies are needed if Europe is to provide mission elements or payloads which need to operate in the lower atmosphere, below $40 \mathrm{~km}$ altitude. For a long-duration surface package, a full suite of subsystems will need to be developed from batteries and power distribution, to data handling, to telecommunications. The development status of these technologies is reviewed elsewhere [19, 20].

Further investment in scientific payloads is also needed to prepare for the Voyage 2050 timeframe. Continued development of planetary radar technologies, though applicable to many planetary missions, is particularly important at Venus because Venus' cloudy atmosphere is opaque to optical imagers. It can be argued that every Venus orbiter should carry a radar imager, just as almost every Mars orbiter has carried an optical imager, to enable follow up of geologically important targets, such as active volcanic and tectonic regions, with ever more capable imagers. Development of more capable and mass efficient radars, with ever increasing spatial and radiometric resolution, and capabilities such as polarimetry and Diffuser, will be invaluable at Venus. Leveraging the investment in Earth observing radar systems for application to Venus will be advantageous. Advanced precursors for heterodyne sub-mm and Doppler LIDAR instrumentation exist in Europe (with JUICE/SWI and MARBLL programmes, respectively, being leading examples); development of Venus versions of these instruments would enable direct measurement of mesospheric winds using Doppler velocimetry.

For atmospheric in situ measurements, a key instrument is a mass spectrometer with getters and cryotraps to isolate and precisely measure the noble gas and light element isotope abundances. These technologies have been developed for Mars (e.g. the SAM instrument on MSL/Curiosity, and the PALOMA instrument originally proposed for ExoMars), but further development to maximise the precision of the measurements and to optimise the development for the thermal environment of Venus balloons and descent probes will be needed. In situ gas chromatography-mass spectrometry characterisation of atmospheric chemistry is another mature field, but further development in 
particular of an aerosol collector system to allow detailed characterisation of cloud particle composition would be valuable.

Specific payload developments for a landing probe should also include surface characterisation instruments - gamma ray and neutron spectrometers, XRF/XRD, Raman instruments for mineralogical identification. High-temperature drilling and sample ingestion systems are not currently proposed for the Venera-D lander included in the strawman mission, but some feasibility studies in this area would be a useful investment. Finally, chemical, meteorological, and seismological sensors using silicon carbide or other $500{ }^{\circ} \mathrm{C}$ temperature semiconductor technology would be key elements of a long-lived lander's payload.

In summary, most of the technologies needed for the proposed Voyage 2050 Venus mission already have high levels of heritage either from Earth or from other planetary missions, but a well-targeted development programme would de-risk mission elements and maximise the science return. Many of the development areas identified would also benefit other space missions and have spin-out potential on Earth.

\section{Conclusions}

As we become aware of Earth's changing climate, and as we discover terrestrial planets in other solar systems, we gain ever more reasons to study the Earth's nearest neighbour and closest sibling, the only Earth-sized planet besides our own that can be reached by our spacecraft.

For the scientific and programmatic reasons outlined in this document, Venus is a compelling target for exploration. The science themes important for Venus research comparative planetology and planetary evolution - are common to all of planetary and exoplanetary science. Many of the payloads required - radar and atmospheric remote sensing, in situ mass spectrometers - are common to mission proposals for many other Solar System targets, as are mission technologies like high rate deep-space telecommunications technologies. Venus-specific technology developments meriting special attention include high-temperature systems and balloons.

Venus is an excellent proving ground for fundamental understanding of geophysical processes of terrestrial planets; an excellent proving ground for techniques of analysis of exoplanets; an indispensable part of our quest to understand the evolution of Earthlike planets. For all these reasons, Venus will be an ever more compelling theme in the coming decades, and we therefore emphatically recommend its inclusion in the Voyage 2050 plan. We suggest that ESA aim to have launched at least two M-class Venus missions by 2050, including the EnVision M5 geophysics orbiter and an in-situ element such as a cloud-level balloon; or an L-class mission combining these elements.

Acknowledgments This manuscript reflects two decades of inputs from the European planetary science community and colleagues across the world, coalescing around ESA's Venus Express mission, ESA's Venus Entry Probe mission study, the European Venus Explorer mission proposals of 2007 and 2010 (led by Eric Chassefière), the EnVision orbiter proposals (led by Richard Ghail), and the Venus Long-life Surface Platform White Paper of 2016. The authors acknowledge the scientific contributions of all the team members of these projects, the engineering contributions of the respective study teams including industrial partners, and the financial support of ESA and national space agencies which have enabled these studies. We thank Juliet Biggs 
for permission to reprint Fig. 2 and Kevin Baines for permission to reprint Fig. 3. C.W. acknowledges funding from the UK Space Agency under grants ST/V001590/1 and ST/P001572/1. R.G. acknowledges funding from the UK Space Agency under grant ST/V00168X/1.

Open Access This article is licensed under a Creative Commons Attribution 4.0 International License, which permits use, sharing, adaptation, distribution and reproduction in any medium or format, as long as you give appropriate credit to the original author(s) and the source, provide a link to the Creative Commons licence, and indicate if changes were made. The images or other third party material in this article are included in the article's Creative Commons licence, unless indicated otherwise in a credit line to the material. If material is not included in the article's Creative Commons licence and your intended use is not permitted by statutory regulation or exceeds the permitted use, you will need to obtain permission directly from the copyright holder. To view a copy of this licence, visit http://creativecommons.org/licenses/by/4.0/.

\section{References}

1. Ghail, R.C. et al, EnVision: understanding why our most Earth-like neighbour is so different. ESA M5 mission proposal (2016). Available at https://arxiv.org/abs/1703.09010

2. Wilson, C.F., et al.: The 2010 European Venus explorer (EVE) mission proposal. Exp. Astron. (2012). https://doi.org/10.1007/s10686-011-9259-9

3. Wilson, C.F. et al., Venus: Key to understanding the evolution of terrestrial planets. White Paper submitted in response to ESA's Call for the definition of science themes for L2/L3 missions in the ESA Science Programme. https://arxiv.org/abs/1703.10961 (2013). Accessed 23 July 2020

4. Marcq, E. et al., Europe returns to Venus. White Paper submitted in response to ESA's Call for the definition of science themes for L2/L3 missions in the ESA Science Programme. http://sci.esa.int/cosmicvision/52030-white-papers-submitted-in-response-to-esas-call-for-science-themes-for-the-12-and-13missions/ (2013b). Accessed 23 July 2020

5. Limaye, S. et al, Venus: a natural planetary laboratory. White Paper submitted in response to ESA's Call for the definition of science themes for L2/L3 missions in the ESA Science Programme. http://sci.esa.int/ cosmic-vision/52030-white-papers-submitted-in-response-to-esas-call-for-science-themes-for-the-12-and13-missions/ (2013). Accessed 23 July 2020

6. Kopparapu, R.K., et al.: Habitable zones around main-sequence stars: new estimates. Astrophys. J. (2013). https://doi.org/10.1088/0004-637X/765/2/131

7. Nakamura, M., et al.: Return to Venus of the Japanese Venus climate orbiter AKATSUKI. Acta Astronautica. (2014). https://doi.org/10.1016/j.actaastro.2013.07.027

8. Turcotte, D.L., et al.: Catastrophic resurfacing and episodic subduction on Venus. Icarus. (1999). https:// doi.org/10.1006/icar.1999.6084

9. Stofan, E.R., Smrekar, S.E.: Large topographic rises, coronae, large flow fields, and large volcanoes on Venus: evidence for mantle plumes? In plates. Plumes Paradigms. (2005). https://doi.org/10.1130/08137-2388-4.841

10. Guest, J.E., Stofan, E.R.: A new view of the stratigraphic history of Venus. Icarus. (1999). https://doi.org/ 10.1006/icar.1999.6091

11. Smrekar, S.E., Davaille, A., Sotin, C.: Venus Interior structure \& dynamics. Space Sci. Rev. (2018). https://doi.org/10.1007/s11214-018-0518-1

12. Smrekar, S.E., et al.: Recent hotspot volcanism on Venus from VIRTIS emissivity data. Science. (2010). https://doi.org/10.1126/science.1186785

13. Marcq, E., et al.: Variations of Sulphur dioxide at the cloud top of Venus's dynamic atmosphere. Nat. Geosci. (2013a). https://doi.org/10.1038/NGEO1650

14. Cottereau, L., et al.: The various contributions in Venus rotation rate and LOD. Astron. Astrophys. (2011). https://doi.org/10.1051/0004-6361/201116606

15. Sparks, R.S.J., et al.: Monitoring volcanoes. Science. (2012). https://doi.org/10.1126/science.1219485

16. Ghail, R.C. et al, VenSAR on EnVision: Taking Earth Observation Radar to Venus. Int. J. Appl. Earth Observ. Geoinf. (2017). https://doi.org/10.1016/j.jag.2017.02.008

17. Mueller, N., et al.: Venus surface thermal emission at $1 \mu \mathrm{m}$ in VIRTIS imaging observations: evidence for variation of crust and mantle differentiation conditions. J. Geophys. Res. (2008). https://doi.org/10. 1029/2008JE003118 
18. Helbert, J. et al, The Venus Emissivity Mapper (VEM) concept, Proc. SPIE 9973, Infrared Remote Sensing and Instrumentation XXIV, 99730R (2016). https://doi.org/10.1117/12.2237568

19. Wilson, C.F. et al, Venus Long-life Surface Package. White paper submitted in response to ESA's Call for New Scientific Ideas. https://arxiv.org/abs/1611.03365 (2016). Accessed 23 July 2020A

20. Kremic, T., et al.: Long-duration Venus lander for seismic and atmospheric science. Planet Space Sci. (2020). https://doi.org/10.1016/j.pss.2020.104961

21. Helbert, J., et al.: Surface brightness variations seen by VIRTIS on Venus Express and implications for the evolution of the Lada Terra region. Venus. Geophysical Research Letters. (2008). https://doi.org/10. 1029/2008GL033609

22. Fegley B. Jr. \& Treiman A.H., Chemistry of Atmosphere-Surface Interactions on Venus and Mars. in Venus and Mars: Atmospheres, Ionospheres, and Solar Wind Interactions. AGU, Geophysical Monograph No. 66, 7-71 (1992)

23. Hashimoto, G.L., Abe, Y. Stabilization of Venus' climate by a chemical-albedo feedback. Earth Planet Sp 52, 197-202 (2000). https://doi.org/10.1186/BF03351628

24. Lebonnois, S., Schubert, G.: The deep atmosphere of Venus and the possible role of density-driven separation of $\mathrm{CO}_{2}$ and $\mathrm{N}_{2}$. Nat. Geosci. 10(p), 473-477 (2017). https://doi.org/10.1038/NGEO2971

25. Gillmann, C., et al.: A consistent picture of early hydrodynamic escape of Venus atmosphere explaining present ne and Ar isotopic ratios and low oxygen atmospheric content. Earth Planet. Sci. Lett. 286, 503513 (2009)

26. Baines, K. H. et al., Experiencing Venus: Clues to the origin, evolution, and chemistry of terrestrial planets via in-situ exploration of our sister world. In Exploring Venus as a Terrestrial Planet. Geophysical Monograph 176, American Geophysical Union, Washington, DC. pp. 171-189 (2007)

27. Chassefière, E., et al.: The evolution of Venus: present state of knowledge and future exploration. Planet Space Sci. (2012). https://doi.org/10.1016/j.pss.2011.04.007

28. Bengtsson, L., et al.: Towards understanding the climate of Venus: applications of terrestrial models to our sister planet. Intl Space Sci Inst Scientific Rep. (2013). https://doi.org/10.1007/978-1-4614-5064-1

29. Andreichikov, B.M. et al., Vega 1 and 2 X-ray radiometer analysis of the Venus cloud aerosol. Kosmicheskie Issledovaniia (ISSN 0023-4206), vol. 25, Sept.-Oct., p. 721-736 (1987)

30. Helbert, J. et al., The VenSpec suite on the ESA EnVision mission to Venus. Proc. SPIE 11128, Infrared Remote Sensing and Instrumentation XXVII, 1112804 (2019). doi: https://doi.org/10.1117/12.2529248

31. Barstow, J.K., et al.: Models of the global cloud structure on Venus derived from Venus express observations. Icarus. (2012). https://doi.org/10.1016/j.icarus.2011.05.018

32. Hensley, S. et al., VISAR : A Next Generation Interferometric Radar for Venus Exploration. Proceedings of the 2015 IEEE 5th Asia-Pacific Conference on Synthetic Aperture Radar (APSAR) (2015). doi: https://doi.org/10.1109/APSAR.2015.7306225

33. Sharpton, V.L. et al., RAVEN - High-resolution Mapping of Venus within a Discovery Mission Budget. AGU Fall Meeting 2009 abstract \#P31D-04 (2009)

34. Blumberg, D.G. et al., MuSAR: a novel SAR mission to Venus. Int. Union of Radio Science General Assembly 2011, abstract J05.1 (2011)

35. Allen, M. et al., The VESPER Mission to Venus. American Astronomical Society, DPS meeting abstract \#48.P08 (1998)

36. Knollenberg and Hunten, The Microphysics of the Clouds of Venus: Results of the Pioneer Venus Particle Size Spectrometer Experiment. J Geophys Res, 85:8039-8058 (1980). doi:https://doi.org/10. 1029/JA085iA13p08039

37. Moroz, V.I.: Estimates of visibility of the surface of Venus from descent probes and balloons. Planet Space Sci. (2002). https://doi.org/10.1016/S0032-0633(01)00128-3

38. Vorontsov, V.A., et al.: Prospective spacecraft for Venus research: Venera-D design. Sol. Syst. Res. (2011). https://doi.org/10.1134/S0038094611070288

39. Mitrofanov, I. et al., Neutron-Activated Gamma Ray Spectrometer (NAGRS) for the Venus Surface and Atmosphere Geochemical Explorer (SAGE) mission. European Planetary Science Congress 2010, abstract \#264 (2010)

40. Clegg, S. et al., Venus Elemental and Mineralogical Camera (VEMCam). European Planetary Science Congress 2019, abstract \#827 (2019)

41. Ghail, R.C., et al.: EnVision: taking the pulse of our twin planet. Exp. Astron. (2012). https://doi.org/10. 1007/s10686-011-9244-3

42. Fujita et al., An overview of Japan's planetary probe mission planning. International Planetary Probe Workshop, Toulouse, France (2012)

43. Nigar, S. et al., ISRO Venus Orbiter Mission. Venus Exploration Analysis Group Meeting, https://www. lpi.usra.edu/vexag/meetings/archive/vexag-17/presentations/Nigar.pdf (2019). Accessed 23 July 2020 
44. Chassefière, E., et al.: European Venus explorer (EVE): an in-situ mission to Venus. Exp. Astron. 23(3), 741-760 (2009). https://doi.org/10.1007/s10686-008-9093-x

45. Gilmore, M. et al., Venus flagship Mission concept study, Venus Exploration Analysis Group Meeting (2019), https://www.hou.usra.edu/meetings/vexag2019/pdf/8019.pdf . Accessed 23 July 2019

46. Hall, J.L., et al.: Technology development for a long duration, mid-cloud level Venus balloon. Adv Spa Res. (2011). https://doi.org/10.1016/j.asr.2011.05.034

Publisher's note Springer Nature remains neutral with regard to jurisdictional claims in published maps and institutional affiliations. 